\title{
Total Synthesis of Malformin C, an Inhibitor of Bleomycin- Induced G2 Arrest
}

\author{
Yasuhiro Kojima, Toshiaki Sunazuka, Kenichiro Nagai, Khachatur Julfakyan, \\ Takashi Fukuda, Hiroshi Tomoda, Satoshi Ōmura
}

Received: April 14, 2008 / Accepted: May 3, 2008

(C) Japan Antibiotics Research Association

\begin{abstract}
Total synthesis of a fungal cyclic peptide, malformin C, recently rediscovered as a G2 checkpoint inhibitor was completed. Our synthesis involved a convergent approach with respect to a linear pentapeptide, cyclization, and oxidative disulfide formation.
\end{abstract}

Keywords total synthesis, malformin C, G2 checkpoint inhibitor, anti-cancer reagent, cyclic peptide, disulfide

\section{Introduction}

Spontaneous and chemical damage to DNA induces signal transduction pathways called checkpoints, which delay cell cycle progression and repair of DNA [1]. DNA damage in normal human cells can be repaired in both the G1 and G2 phases. In contrast, most cancer cells can restore DNA damage only in the $\mathrm{G} 2$ phase due to mutations in genes for the G1 checkpoint. Therefore, an inhibitor of the G2 checkpoint in cancer cells is expected to be a selective potentiator of DNA-damaging agents and thus useful for the treatment of cancer.

Recently, malformins A1 and C (Fig. 1), isolated from the culture broth of Aspergillus niger FKI-2342, were found to abrogate the bleomycin-induced G2 arrest in

S. Ōmura (Corresponding author), Y. Kojima, T. Sunazuka, K. Nagai, K. Julfakyan: Kitasato Institute for Life Sciences and Graduate School of Infection Control Sciences, Kitasato University, 5-9-1 Shirokane, Minato-ku, Tokyo 108-8641, Japan, E-mail: omuras@insti.kitasato-u.ac.jp

T. Fukuda, H. Tomoda: School of Pharmacy, Kitasato University, 5-9-1 Shirokane, Minato-ku, Tokyo 108-8641, Japan
Jurkat cells with $\mathrm{IC}_{50}$ values of $480 \mathrm{nM}$ and $0.9 \mathrm{nM}$, respectively [2]. Malformin A1 is a bicyclic pentapeptide containing one L-isoleucine, one D-leucine, one L-valine, and two D-cysteines, while malformin $\mathrm{C}$ has L-leucine instead of L-isoleucine. Interestingly, this slight structural difference of the side-chains has an influence on the inhibitory activity. Consequently, malformin $\mathrm{C}$ is a promising candidate as a potentiator of anti-cancer agents. Although malformins are also known to show a variety of activities such as inducing root curvatures and malformations in plants, antibacterial activity and enhanced fibrinolytic activity [3 6], the interesting bioactivity of malformin $\mathrm{C}$, as well as its bicyclic structure with a disulfide-bond bridge, prompted us to study the synthesis of this class of cyclic peptides. Herein, we describe a synthesis of malformin C.

\section{Results and Discussion}

We planned to undertake the synthesis of malformin C via a convergent approach, involving preparation and coupling of tripeptide (1) and dipeptide (2) to allow creation of linear
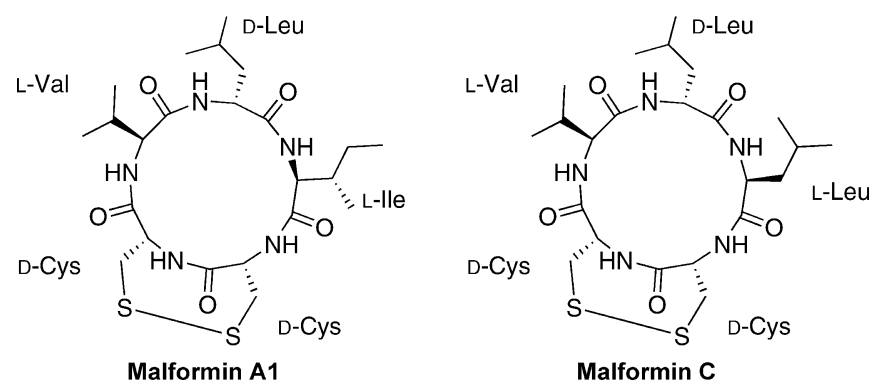

Fig. 1 Structures of malformins A1 and C. 
pentapeptide (3), which should undergo cyclization and oxidative disulfide formation (Scheme 1). The less hindered amide bond between D-cysteine and L-valine was chosen for cyclization because the cyclization position between Dleucine and L-isoleucine has already been investigated in the stepwise synthesis of malformin A1 [7].

Total synthesis of malformin C is shown in Scheme 2. Coupling of L-leucine benzyl ester (4) and Boc-D-leucine, followed by removal of the Boc group with $4.0 \mathrm{~N}$ $\mathrm{HCl}$ /dioxane provided dipeptide (5), which was subjected to condensation of Fmoc-L-valine. Hydrogenolysis of the benzyl group proceeded in a catalytic amount of $\mathrm{Pd}(\mathrm{OH})_{2}$ at $40^{\circ} \mathrm{C}$ to give tripeptide 1 . Heating was necessary to dissolve the Fmoc protected tripeptide in ethyl acetate. D-S-Tritylcystein allyl ester (7) was prepared from commercially available Fmoc-D-S-tritylcystein (6) by allylation of the carboxylic acid, followed by deprotection of the Fmoc group. After coupling of $\mathbf{6}$ and 7, dipeptide $\mathbf{2}$ was obtained by deprotection of the Fmoc group with piperidine. Coupling of $\mathbf{1}$ and $\mathbf{2}$ using HBTU/HOBt

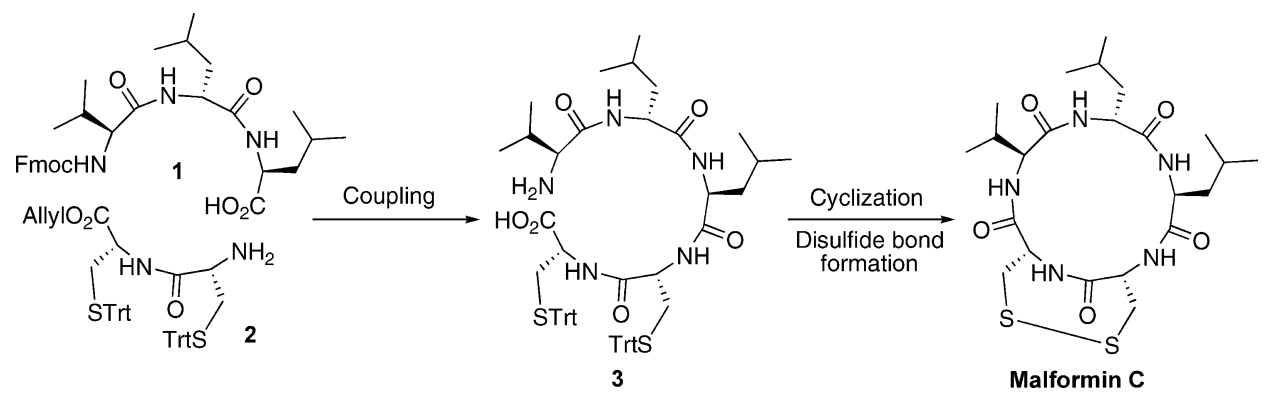

Scheme 1 Plan for synthesis of malformin C

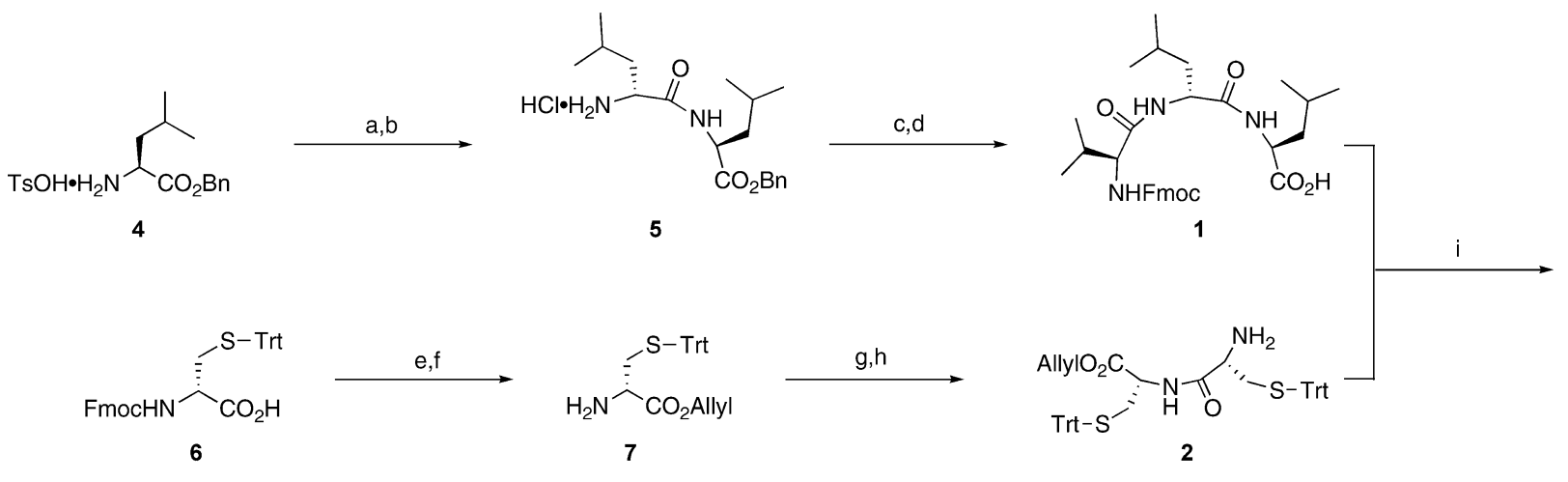

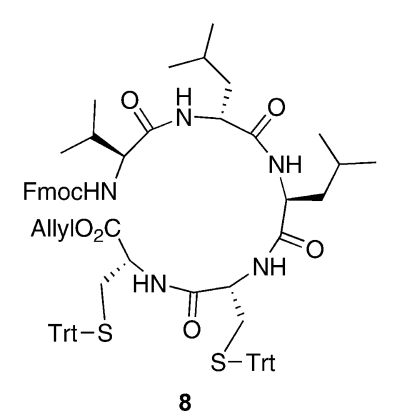

8

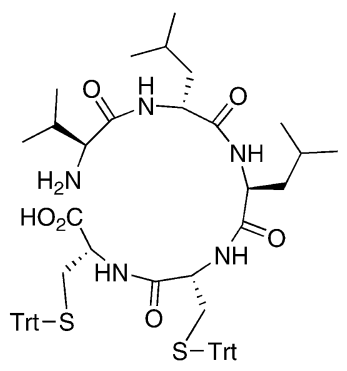

3

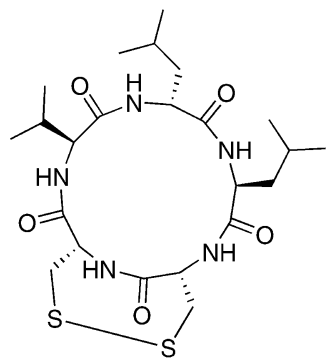

Malformin C

Scheme 2 Synthesis of malformin $C$

a) Boc-D-Leu-OH, EDCl, HOBt, DIPEA, $\mathrm{CH}_{2} \mathrm{Cl}_{2}, \mathrm{rt}, 98 \%$; b) $4.0 \mathrm{~N} \mathrm{HCl} /$ dioxane, $0^{\circ} \mathrm{C}$; c) Fmoc-L-Val-OH, EDCl, $\mathrm{HOBt} \mathrm{DIPEA} \mathrm{CH}_{2} \mathrm{Cl}_{2} / \mathrm{DMF}$ (4/1), rt; d) $\mathrm{H}_{2}, \mathrm{Pd}(\mathrm{OH})_{2}$, EtOAc, $40^{\circ} \mathrm{C}, 86 \%$ in 3 steps; e) $\mathrm{Cs}_{2} \mathrm{CO}_{3}, \mathrm{AllylBr}, \mathrm{DMF}$, rt; f) piperidine, $\mathrm{CH}_{2} \mathrm{Cl}_{2}, 0^{\circ} \mathrm{C}, 93 \%$ in 2 steps; g) Fmoc-DCys(Trt)-OH 6, EDCl, HOBt, DIPEA, $\mathrm{CH}_{2} \mathrm{Cl}_{2}, \mathrm{rt}$; h) piperidine, $\mathrm{CH}_{2} \mathrm{Cl}_{2}, 0^{\circ} \mathrm{C}, 72 \%$ in 2 steps; i) $\mathrm{HBTU}, \mathrm{HOBt}, \mathrm{NMM}^{\circ} \mathrm{CH}_{2} \mathrm{Cl}_{2} / \mathrm{DMF}(4 / 1), \mathrm{rt}, 93 \%$;

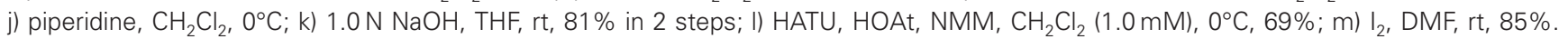
DIPEA=diisopropylethylamine,$\quad \mathrm{EDCl}=1$-ethyl-3-(3-dimethylaminopropyl)carbodiimide hydrochloride, HATU=O-(7-azabenzotriazol-1-yl)$N, N, N^{\prime}, N^{\prime}$-tetramethyluronium hexafluorophosphate, HBTU=O-(benzotriazol-1-yl)- $N, N_{,} N^{\prime}, N^{\prime}$-tetramethyluronium hexafluorophosphate, $\mathrm{HOAt}=1$-hydroxy-7-azabenzotriazole, $\mathrm{HOBt}=1$-hydroxybenzotriazole, $\mathrm{NMM}=\mathrm{N}$-methylmorphorine. 
provided linear peptide (8) in $93 \%$ yield. Cyclization precursor 3 was obtained by removal of the Fmoc group, followed by hydrolysis of the Allyl group with aq $\mathrm{NaOH}$. In the deprotection of the Allyl group, palladium-catalyzed reaction with dimedone resulted in poor yield. With linear peptide 3 in hand, we examined the effect of conditions on cyclization. After several attempts, combination of HATU and HOAt under high dilution conditions $(1.0 \mathrm{mM})$ at $0{ }^{\circ} \mathrm{C}$ afforded the cyclic peptide in $69 \%$ yield. Other coupling reagents such as HBTU/HOBt and PyBop reduced the yields (44 and 34\%). Finally, oxidative disulfide formation with iodine in DMF smoothly proceeded, resulting in malformin $\mathrm{C}$ in $85 \%$ yield after silica gel chromatography. Its yield was reduced to $26 \%$ when $10 \% \mathrm{MeOH} / \mathrm{CH}_{2} \mathrm{Cl}_{2}$ was used as a solvent system. Spectra data of the synthetic malformin $\mathrm{C}$ were found to be identical to those of the natural product.

In conclusion, we have demonstrated a synthesis of malformin C, a fungal cyclic peptide, in 9 steps and $30 \%$ overall yield. The results could be exploited to generate malformin derivatives for elucidation of the structureactivity relationships. Further studies on in vivo activities and solid phase synthesis of malformin derivatives are in progress.

\section{Experimental}

Reagents were purchased at highest commercial quality and used without further purification, unless otherwise specified. Reactions were monitored by TLC using Merck $\mathrm{F} 60_{254}$ silica gel plates. Spots were visualized with UV light $(254 \mathrm{~nm})$ and stained with phosphomolybdic acid and ninhydrin. Silica gel chromatography was performed on a Merck Kieselgel 60 (Art. 1.09385).

FT-IR spectra were recorded in $\mathrm{KBr}$ pellets on a Horiba FT-210 spectrometer. Mass spectra were recorded on a JEOL JMS-700V Mass Spectrometer. ${ }^{1} \mathrm{H}-\mathrm{NMR}$ spectra were recorded on a JEOL JNM-EX270 spectrometer in $\mathrm{CDCl}_{3}$ or pyridine- $d_{5} .{ }^{1} \mathrm{H}-\mathrm{NMR}$ spectral data are reported as follows: chemical shifts relative to $\mathrm{CHCl}_{3}(7.26 \mathrm{ppm})$ or pyridine $(8.71 \mathrm{ppm})$, integration, multiplicity $(\mathrm{s}=$ singlet, $\mathrm{d}=$ doublet, $\mathrm{t}=$ triplet, $\mathrm{q}=$ quartet, $\mathrm{m}=$ multiplet, $\mathrm{br}=$ broad) and coupling constant. Optical rotation was obtained with a JASCO DIP-370 polarimeter. ${ }^{13} \mathrm{C}-\mathrm{NMR}$ spectral data are reported in ppm relative to $\mathrm{CHCl}_{3}(77.0 \mathrm{ppm})$ or pyridine (135.5 ppm). Melting points were measured with a Yanaco micromelting point apparatus.

\section{Benzyl (N-tert-Butoxycarbonyl-D-leucinyl)-L-leucinate}

To a solution of $4(3.27 \mathrm{~g}, 9.63 \mathrm{mmol})$ in $\mathrm{CH}_{2} \mathrm{Cl}_{2}(80 \mathrm{ml})$ were added $N$-Boc-D-Leu $(2.0 \mathrm{~g}, 8.02 \mathrm{mmol})$, DIPEA $(3.07 \mathrm{ml}, 17.6 \mathrm{~mol})$, HOBt $(2.60 \mathrm{~g}, 19.3 \mathrm{mmol})$ and $\mathrm{EDCl}$ $(1.85 \mathrm{~g}, 9.63 \mathrm{mmol})$ at $0^{\circ} \mathrm{C}$, and the mixture was stirred at room temperature for 2 hours. The reaction was quenched with $\mathrm{H}_{2} \mathrm{O}$, and the resulting mixture was extracted with $\mathrm{CHCl}_{3}$. The organic extracts were washed with saturated aq $\mathrm{NH}_{4} \mathrm{Cl}$, saturated aq $\mathrm{NaHCO}_{3}$ and brine, dried over $\mathrm{Na}_{2} \mathrm{SO}_{4}$, filtered, and concentrated. The residue was purified by flash column chromatography on silica gel (hexane/EtOAc $=5 / 1$ to $4 / 1)$ to give the dipeptide $(3.40 \mathrm{~g}$, $98 \%)$ as a white solid. $\mathrm{mp} 91{ }^{\circ} \mathrm{C} ;[\alpha]_{\mathrm{D}}^{22}+22.4(\mathrm{c} 1.0$, $\mathrm{CHCl}_{3}$ ); IR (KBr) cm ${ }^{-1}$; 3332, 2958, 1725, 1685, 1652, $1542,1523,1168,752,701 ;{ }^{1} \mathrm{H}-\mathrm{NMR}\left(270 \mathrm{MHz}, \mathrm{CDCl}_{3}\right) \delta$ $7.32 \sim 7.19(5 \mathrm{H}, \mathrm{m}), 6.63(1 \mathrm{H}, \mathrm{s}), 5.07(2 \mathrm{H}, \mathrm{dd}, J=8.2$, $10.7 \mathrm{~Hz}), 4.85(1 \mathrm{H}, \mathrm{s}), 4.57(1 \mathrm{H}, \mathrm{dt}, J=4.8,8.4 \mathrm{~Hz})$, $4.08 \sim 4.00(1 \mathrm{H}, \mathrm{m}), 1.60 \sim 1.38(6 \mathrm{H}, \mathrm{m}), 1.36(9 \mathrm{H}, \mathrm{s})$, $0.84 \sim 0.82(12 \mathrm{H}, \mathrm{m}) ;{ }^{13} \mathrm{C}-\mathrm{NMR}\left(67.2 \mathrm{MHz}, \mathrm{CDCl}_{3}\right) \delta$ $172.5,172.4,155.5,135.2,128.2,128.0,127.8,79.4,66.5$, 52.8, 50.5, 40.9, 40.7, 28.0, 24.5, 22.6, 21.7, 21.4; MS (FAB) $m / z 435.2859[\mathrm{M}+\mathrm{H}]^{+}$(calcd for $\mathrm{C}_{24} \mathrm{H}_{39} \mathrm{O}_{5} \mathrm{~N}_{2}$ : $435.2859[\mathrm{M}+\mathrm{H}])$.

\section{Benzyl D-Leucinyl-L-leucinate Hydrochloride (5)}

To a solution of dipeptide $(3.40 \mathrm{~g}, 7.82 \mathrm{mmol})$ was added $4.0 \mathrm{~N} \mathrm{HCl} /$ dioxane $(39 \mathrm{ml})$ at $0^{\circ} \mathrm{C}$, and the mixture was stirred at same temperature for 2 hours. The reaction mixture was concentrated to give $\mathbf{5}$ as a white solid. The title compound was used for subsequent reaction without further purification.

\section{Benzyl N-9-Fluorenylmethyloxycarbonyl-L-valinyl- D-leucinyl-L-leucinate}

To a solution of 5 (7.82 mmol) in $\mathrm{CH}_{2} \mathrm{Cl}_{2} / \mathrm{DMF}(4 / 1,78 \mathrm{ml})$ were added $N$-Fmoc-L-Val $(3.19 \mathrm{~g}, 9.39 \mathrm{mmol})$, DIPEA $(8.18 \mathrm{ml}, 46.9 \mathrm{mmol})$, HOBt $(2.11 \mathrm{~g}, 10.6 \mathrm{mmol})$ and $\mathrm{EDCl}$ $(3.0 \mathrm{~g}, 10.6 \mathrm{mmol})$ at $0^{\circ} \mathrm{C}$, and the mixture was stirred at room temperature for 4 hours. The reaction was quenched with $\mathrm{H}_{2} \mathrm{O}$, and the resulting mixture was extracted with $\mathrm{CHCl}_{3}$. The organic extracts were washed with saturated aq $\mathrm{NH}_{4} \mathrm{Cl}$, saturated aq $\mathrm{NaHCO}_{3}$ and brine, dried over $\mathrm{Na}_{2} \mathrm{SO}_{4}$, filtered, and concentrated. The residue was recrystallized from $\mathrm{CHCl}_{3}$ and hexane to give the tripeptide (5.30 g, quant.) as a white solid. mp $145 \sim 150^{\circ} \mathrm{C} ;[\alpha]_{\mathrm{D}}^{23}$ +16.2 (c 1.0, $\left.\mathrm{CHCl}_{3}\right)$; IR (KBr) cm ${ }^{-1} ; 3299,2956,1720$, 1691, 1643, 1535, 1247, 1029, 732; ${ }^{1} \mathrm{H}-\mathrm{NMR}(270 \mathrm{MHz}$, $\left.\mathrm{CDCl}_{3}\right) \delta 7.75(2 \mathrm{H}, \mathrm{d}, J=7.6 \mathrm{~Hz}), 7.57(2 \mathrm{H}, \mathrm{d}, J=7.3 \mathrm{~Hz})$, $7.42 \sim 7.30(9 \mathrm{H}, \mathrm{m}), 6.80(1 \mathrm{H}, \mathrm{d}, J=6,9 \mathrm{~Hz}), 6.39(1 \mathrm{H}, \mathrm{d}$, $J=7.9 \mathrm{~Hz}), 5.37(1 \mathrm{H}, \mathrm{d}, J=7.9 \mathrm{~Hz}), 5.08(2 \mathrm{H}, \mathrm{dd}, J=10.0$, $14.5 \mathrm{~Hz}), 4.62 \sim 4.42(3 \mathrm{H}, \mathrm{m}), 4.31(1 \mathrm{H}, \mathrm{t}, J=8.6 \mathrm{~Hz}), 4.20$ $(1 \mathrm{H}, \mathrm{t}, J=6.8 \mathrm{~Hz}), 3.87(1 \mathrm{H}, \mathrm{t}, J=7.1 \mathrm{~Hz}), 2.15(1 \mathrm{H}, \mathrm{m})$, $1.75 \sim 1.47(6 \mathrm{H}, \mathrm{m}), 0.93(6 \mathrm{H}, \mathrm{d}, J=6.3 \mathrm{~Hz}), 0.91(6 \mathrm{H}, \mathrm{d}$, 
$J=5.8 \mathrm{~Hz}), 0.84(6 \mathrm{H}, \mathrm{d}, J=5.3 \mathrm{~Hz}) ;{ }^{13} \mathrm{C}-\mathrm{NMR}(67.5 \mathrm{MHz}$, $\left.\mathrm{CDCl}_{3}\right) \delta 172.6,171.8,169.2,156.6,143.8,141.2,135.4$, $128.5,128.3,127.7,127.1,125.0,120.0,67.0,53.1,51.5$, 50.9, 47.1, 42.0, 40.5, 30.7, 24.8, 24.2, 23.1, 22.7, 21.8, 21.3, 19.2, 18.0; MS (FAB) $m / z 656.3711[\mathrm{M}+\mathrm{H}]^{+}$(calcd for $\left.\mathrm{C}_{39} \mathrm{H}_{50} \mathrm{O}_{6} \mathrm{~N}_{3}: 656.3700[\mathrm{M}+\mathrm{H}]\right)$.

\section{$N$-9-Fluorenylmethyloxycarbonyl-L-valinyl-D-leucinyl- L-leucine (1)}

$\mathrm{Pd}(\mathrm{OH})_{2}(20 \% \mathrm{Pd}$ on carbon) $(21.3 \mathrm{mg}, 30.4 \mu \mathrm{mol})$ was activated in EtOAc $(3.0 \mathrm{ml})$ under $\mathrm{H}_{2}$ atmosphere at room temperature for 30 minutes. The tripeptide $(99.7 \mathrm{mg}$, $152 \mu \mathrm{mol})$ was added to the mixture. After stirring at $40^{\circ} \mathrm{C}$ for 6 hours, the reaction mixture was filtered through a pad of Celite, and the filtrate was concentrated. The residue was purified by flash column chromatography on silica gel $\left(\mathrm{CHCl}_{3}\right.$ to $\left.\mathrm{CHCl}_{3} / \mathrm{MeOH}=10 / 1\right)$ to give $1(74.2 \mathrm{mg}, 86 \%)$ as a light yellow solid. mp $105^{\circ} \mathrm{C} ;[\alpha]_{\mathrm{D}}^{22}+6.8(c$ 1.0, $\mathrm{CHCl}_{3}$ ); IR (KBr) cm ${ }^{-1} ; 3288,2958,1712,1668,1650$, 1573, 1515, 1230, 759, 740; ${ }^{1} \mathrm{H}-\mathrm{NMR}(270 \mathrm{MHz}$, Pyridine$\left.d_{5}\right) \delta 9.65(1 \mathrm{H}, \mathrm{d}, J=8.3 \mathrm{~Hz}), 9.00(1 \mathrm{H}, \mathrm{d}, J=7.3 \mathrm{~Hz}), 8.65$ $(1 \mathrm{H}, \mathrm{d}, J=8.9 \mathrm{~Hz}), 7.71(2 \mathrm{H}, \mathrm{d}, J=7.6 \mathrm{~Hz}), 7.65(2 \mathrm{H}, \mathrm{d}$, $J=7.6 \mathrm{~Hz}), 7.40 \sim 7.16(4 \mathrm{H}, \mathrm{m}), 5.24 \sim 5.12(2 \mathrm{H}, \mathrm{m}), 4.72$ $(1 \mathrm{H}, \mathrm{dd}, J=6.9,10.2 \mathrm{~Hz}), 4.46(2 \mathrm{H}, \mathrm{dd}, J=7.3,15.8 \mathrm{~Hz})$, $4.30(1 \mathrm{H}, \mathrm{t}, J=6.7 \mathrm{~Hz}), 2.49 \sim 2.36(1 \mathrm{H}, \mathrm{m}), 2.12 \sim 1.83$ $(6 \mathrm{H}, \mathrm{m}), 1.16(3 \mathrm{H}, \mathrm{d}, J=6.9 \mathrm{~Hz}), 1.11(3 \mathrm{H}, \mathrm{d}, J=6.6 \mathrm{~Hz})$, $0.90 \sim 0.84(12 \mathrm{H}, \mathrm{m}) ;{ }^{13} \mathrm{C}-\mathrm{NMR}\left(67.2 \mathrm{MHz}\right.$, Pyridine- $\left.d_{5}\right) \delta$ $173.3,173.1,158.1,144.7,141.9,128.3,127.7,126.0$, $125.9,120.7,120.6,80.0,67.2,62.8,52.6,48.0,41.9,41.5$, $31.0,25.5,25.4,23.7,23.4,22.4,21.5,20.1,19.6$; MS (FAB) $m / z 588.3059[\mathrm{M}+\mathrm{Na}]^{+}$(calcd for $\mathrm{C}_{32} \mathrm{H}_{43} \mathrm{O}_{6} \mathrm{~N}_{3} \mathrm{Na}$ : $588.3050[\mathrm{M}+\mathrm{Na}])$.

\section{Allyl N-9-Fluorenylmethyloxycarbonyl-D-S- tritylcysteinate}

To a solution of 6 (586 mg, $1.00 \mathrm{mmol})$ in $\mathrm{H}_{2} \mathrm{O} / \mathrm{MeOH}(1 / 1$, $10 \mathrm{ml})$ was added $\mathrm{Cs}_{2} \mathrm{CO}_{3}(196 \mathrm{mg}, 0.6 \mathrm{mmol})$ at $0^{\circ} \mathrm{C}$. After stirring at room temperature for 1 hour, the reaction mixture was concentrated. The residue was dissolved in DMF $(2.0 \mathrm{ml})$, and then allyl bromide $(173 \mu 1,2.0 \mathrm{mmol})$ was added at $0^{\circ} \mathrm{C}$. The mixture was stirred at room temperature for 9.5 hours. The reaction mixture was diluted with EtOAc and washed with $\mathrm{H}_{2} \mathrm{O}$. The organic layer was dried over $\mathrm{Na}_{2} \mathrm{SO}_{4}$, filtered, and concentrated. The residue was purified by flash column chromatography on silica gel (hexane/EtOAc $=5 / 1$ to $3 / 1)$ to give the allyl ester $(624 \mathrm{mg}$, quant.) as a white solid. mp $55^{\circ} \mathrm{C} ;[\alpha]_{\mathrm{D}}^{26}-14.3$ (c 1.0, $\mathrm{CHCl}_{3}$ ); IR (KBr) cm $\mathrm{cm}^{-1}$; 3415, 3060, 1727, 1509, 1446, 1334, 1186, 1051, 742, 701; ${ }^{1} \mathrm{H}-\mathrm{NMR}\left(270 \mathrm{MHz}, \mathrm{CDCl}_{3}\right) \delta$ $7.77(2 \mathrm{H}, \mathrm{d}, J=7.3 \mathrm{~Hz}), 7.61(2 \mathrm{H}, \mathrm{d}, J=7.3 \mathrm{~Hz}), 7.42 \sim 7.18$ $(19 \mathrm{H}, \mathrm{m}), 5.88(1 \mathrm{H}, \mathrm{m}), 5.34 \sim 5.23(2 \mathrm{H}, \mathrm{m}), 4.62(2 \mathrm{H}, \mathrm{d}$,
$J=5.3 \mathrm{~Hz}), 4.43 \sim 4.35(3 \mathrm{H}, \mathrm{m}), 4.23(1 \mathrm{H}, \mathrm{t}, J=7.1 \mathrm{~Hz})$, $2.68(1 \mathrm{H}, \mathrm{dd}, J=6.3,12.5 \mathrm{~Hz}), 2.65(1 \mathrm{H}, \mathrm{dd}, J=4.6$, $12.5 \mathrm{~Hz}) ;{ }^{13} \mathrm{C}-\mathrm{NMR}\left(67.2 \mathrm{MHz}, \mathrm{CDCl}_{3}\right) \delta 170.1,155.5$, $144.2,144.1,143.7,143.6,141.1,131.3,129.4,127.9$, 127.6, 127.0, 126.8, 125.0, 119.8, 118.5, 66.9, 66.0, 60.2, 52.9, 46.9, 33.9; MS (FAB) $m / z 648.2196[\mathrm{M}+\mathrm{Na}]^{+}$(calcd for $\mathrm{C}_{40} \mathrm{H}_{35} \mathrm{O}_{4} \mathrm{NSNa} 648.2185[\mathrm{M}+\mathrm{Na}]$ ).

\section{Allyl D-S-Tritylcysteinate (7)}

A mixture of the allyl ester $(297 \mathrm{mg}, 475 \mu \mathrm{mol})$ in piperidine $/ \mathrm{CH}_{2} \mathrm{Cl}_{2}(1 / 4,4.8 \mathrm{ml})$ was stirred at $0^{\circ} \mathrm{C}$ for 3 hours. The reaction was quenched with $\mathrm{H}_{2} \mathrm{O}$, and the resulting mixture was extracted with $\mathrm{CHCl}_{3}$. The organic extracts were washed with saturated aq $\mathrm{NH}_{4} \mathrm{Cl}$ and brine, dried over $\mathrm{Na}_{2} \mathrm{SO}_{4}$, filtered and concentrated. The residue was purified by flash column chromatography on silica gel (hexane/EtOAc $=10 / 1$ to $1 / 1)$ to give $7(178 \mathrm{mg}, 93 \%$ ) as a yellow oil; $[\alpha]_{\mathrm{D}}^{21}-44.7\left(\right.$ c $\left.1.0, \mathrm{CHCl}_{3}\right)$; IR $(\mathrm{KBr}) \mathrm{cm}^{-1}$; $3058,1735,1488,1444,1174,742,700 ;{ }^{1} \mathrm{H}-\mathrm{NMR}$ $\left(270 \mathrm{MHz}, \mathrm{CDCl}_{3}\right) \delta 7.45 \sim 7.18(15 \mathrm{H}, \mathrm{m}), 5.93 \sim 5.78(1 \mathrm{H}$, $\mathrm{m}), 5.27(1 \mathrm{H}, \mathrm{dd}, J=1.3,13.7 \mathrm{~Hz}), 5.22(1 \mathrm{H}, \mathrm{dd}, J=1.3$, $6.3 \mathrm{~Hz}), 4.56(2 \mathrm{H}, \mathrm{d}, J=5.6 \mathrm{~Hz}), 3.24(1 \mathrm{H}, \mathrm{dd}, J=5.0$, $7.6 \mathrm{~Hz}), 2.62(1 \mathrm{H}, \mathrm{dd}, J=5.0,12.6 \mathrm{~Hz}), 2.54(1 \mathrm{H}, \mathrm{dd}$, $J=7.6,12.6 \mathrm{~Hz}) ;{ }^{13} \mathrm{C}-\mathrm{NMR}\left(67.2 \mathrm{MHz}, \mathrm{CDCl}_{3}\right) \delta 173.2$, 144.4, 131.6, 129.4, 127.8, 126.6, 118.3, 66.7, 65.5, 53.7, 36.7; MS (FAB) $m / z$ 426.1500 $[\mathrm{M}+\mathrm{Na}]^{+}$(calcd for $\mathrm{C}_{25} \mathrm{H}_{25} \mathrm{O}_{2} \mathrm{NSNa}$ : $\left.426.1504[\mathrm{M}+\mathrm{Na}]\right)$.

\section{Allyl N-9-Fluorenylmethyloxycarbonyl-D-S- tritylcysteinyl-D-S-tritylcysteinate}

To a solution of $7(38.7 \mathrm{mg}, 95.9 \mu \mathrm{mol})$ in $\mathrm{CH}_{2} \mathrm{Cl}_{2}(0.96 \mathrm{ml})$ were added $N$-Fmoc-D-( $S$-Trt)-Cys $(67.4 \mathrm{mg}, 115 \mu \mathrm{mol})$, DIPEA $(100 \mathrm{ml}, 575 \mu \mathrm{mol})$, HOBt $(25.9 \mathrm{mg}, 192 \mu \mathrm{mol})$ and $\mathrm{EDCl}(36.8 \mathrm{mg}, 192 \mu \mathrm{mol})$ at $0^{\circ} \mathrm{C}$, and the mixture was stirred at room temperature for 4 hours. The reaction was quenched with $\mathrm{H}_{2} \mathrm{O}$, and the resulting mixture was extracted with $\mathrm{CHCl}_{3}$. The organic extracts were washed with saturated aq $\mathrm{NH}_{4} \mathrm{Cl}$, saturated aq $\mathrm{NaHCO}_{3}$ and brine, dried over $\mathrm{Na}_{2} \mathrm{SO}_{4}$, filtered, and concentrated. The residue was purified by flash column chromatography on silica gel (hexane/EtOAc $=10 / 1)$ to give the dipeptide $(82.9 \mathrm{mg}, 89 \%)$ as a white solid. $\mathrm{mp} 74^{\circ} \mathrm{C}$; $[\alpha]_{\mathrm{D}}^{23}-6.6\left(\mathrm{c} 1.0, \mathrm{CHCl}_{3}\right)$; IR $(\mathrm{KBr}) \mathrm{cm}^{-1}$; 3390, 3054, 1737, 1681, 1488, 1444, 742, $700 ;{ }^{1} \mathrm{H}-\mathrm{NMR}\left(270 \mathrm{MHz}, \mathrm{CDCl}_{3}\right) \delta 7.77(2 \mathrm{H}, \mathrm{t}, J=6.6 \mathrm{~Hz})$, $7.55(2 \mathrm{H}, \mathrm{s}), 7.44 \sim 7.13(34 \mathrm{H}, \mathrm{m}), 6.33(1 \mathrm{H}, \mathrm{d}, J=7.6 \mathrm{~Hz})$, $5.90 \sim 5.73(1 \mathrm{H}, \mathrm{m}), 5.27(1 \mathrm{H}, \mathrm{dd}, J=1.3,13.7 \mathrm{~Hz}), 5.18$ $(1 \mathrm{H}, \mathrm{dd}, J=1.3,6.3 \mathrm{~Hz}), 4.98(1 \mathrm{H}, \mathrm{d}, J=6.3 \mathrm{~Hz}), 4.54(1 \mathrm{H}$, d, $J=5.0 \mathrm{~Hz}), 4.47 \sim 4.29(3 \mathrm{H}, \mathrm{m}), 4.18(1 \mathrm{H}, \mathrm{t}, J=7.1 \mathrm{~Hz})$, $3.76(1 \mathrm{H}, \mathrm{dd}, J=5.8,7.3 \mathrm{~Hz}), 2.65(2 \mathrm{H}, \mathrm{dd}, J=6.0,8.1 \mathrm{~Hz})$, $2.58(2 \mathrm{H}, \mathrm{t}, J=5.0 \mathrm{~Hz}) ;{ }^{13} \mathrm{C}-\mathrm{NMR}\left(67.2 \mathrm{MHz}, \mathrm{CDCl}_{3}\right) \delta$ $169.7,169.3,155.7,144.3,144.1,143.8,143.6,141.2$, 
$131.2,129.5,129.3,128.0,127.9,127.7,127.0,126.8$ 125.0, 119.9, 118.8, 67.3, 66.7, 66.2, 53.8, 51.3, 47.0, 33.5; MS (FAB) $m / z \quad 971.3588 \quad[\mathrm{M}+\mathrm{H}]^{+} \quad$ (calcd for $\left.\mathrm{C}_{62} \mathrm{H}_{55} \mathrm{O}_{5} \mathrm{~N}_{2} \mathrm{~S}_{2}: 971.3552[\mathrm{M}+\mathrm{H}]\right)$.

\section{Allyl D-S-Tritylcysteinyl-D- $S$-tritylcysteinate (2)}

A mixture of the dipeptide $(67.5 \mathrm{mg}, 69.5 \mu \mathrm{mol})$ in piperidine $/ \mathrm{CH}_{2} \mathrm{Cl}_{2}(1 / 4,0.70 \mathrm{ml})$ was stirred at $0^{\circ} \mathrm{C}$ for 1.5 hours. The reaction was quenched with $\mathrm{H}_{2} \mathrm{O}$, and the resulting mixture was extracted with $\mathrm{CHCl}_{3}$. The organic extracts were washed with saturated aq $\mathrm{NH}_{4} \mathrm{Cl}$ and brine, dried over $\mathrm{Na}_{2} \mathrm{SO}_{4}$, filtered, and concentrated. The residue was purified by flash column chromatography on silica gel (hexane/EtOAc $=5 / 1$ to $1 / 1)$ to give $2(42.1 \mathrm{mg}, 81 \%)$ as a white solid. mp $60 \sim 63^{\circ} \mathrm{C}$; $[\alpha]_{\mathrm{D}}^{21}-20.6\left(c 1.0, \mathrm{CHCl}_{3}\right)$; IR $(\mathrm{KBr}) \mathrm{cm}^{-1}$; 3374, 3014, 1743, 1673, 1492, 1432, 1182, 742, 692, 669; ${ }^{1} \mathrm{H}-\mathrm{NMR}\left(270 \mathrm{MHz}, \mathrm{CDCl}_{3}\right) \delta 7.54 \sim 7.16$ $(30 \mathrm{H}, \mathrm{m}), 5.89 \sim 5.75(1 \mathrm{H}, \mathrm{m}), 5.28 \sim 5.15(2 \mathrm{H}, \mathrm{m}), 4.55$ $(2 \mathrm{H}, \mathrm{d}, J=5.6 \mathrm{~Hz}), 4.43(1 \mathrm{H}, \mathrm{dt}, J=5.5,8.0 \mathrm{~Hz}), 2.95(1 \mathrm{H}$, $\mathrm{dd}, J=4.0,8.9 \mathrm{~Hz}), 2.75(1 \mathrm{H}, \mathrm{dd}, J=4.0,13.2 \mathrm{~Hz})$, $2.62 \sim 2.53(3 \mathrm{H}, \mathrm{m}) ;{ }^{13} \mathrm{C}-\mathrm{NMR}\left(67.2 \mathrm{MHz}, \mathrm{CDCl}_{3}\right) \delta 172.5$, $169.6,144.3,144.0,131.2,129.3,129.2,127.7,126.5$, 118.3, 66.7, 66.3, 65.8, 53.6, 50.7, 36.9, 33.6; MS (FAB) $m / z 749.2853[\mathrm{M}+\mathrm{H}]^{+}$(calcd for $\mathrm{C}_{47} \mathrm{H}_{45} \mathrm{O}_{3} \mathrm{~N}_{2} \mathrm{~S}_{2}: 749.2872$ $[\mathrm{M}+\mathrm{H}])$.

\section{Allyl N-9-Fluorenylmethyloxycarbonyl-L-valinyl- D-leucinyl-L-leucinyl-D- $S$-tritylcysteinyl-D- $S$ - tritylcysteinate (8)}

To a solution of $\mathbf{1}(31.8 \mathrm{mg}, 562 \mu \mathrm{mol})$ and $\mathbf{2}(35.1 \mathrm{mg}$, $469 \mu \mathrm{mol})$ in $\mathrm{CH}_{2} \mathrm{Cl}_{2} / \mathrm{DMF}(4 / 1,0.47 \mathrm{ml})$ were added NMM (13.6 $\mu \mathrm{l}, 122 \mu \mathrm{mol})$, HOBt $(9.3 \mathrm{mg}, 609 \mu \mathrm{mol})$ and HBTU $(23.1 \mathrm{mg}, 609 \mu \mathrm{mol})$ at $0^{\circ} \mathrm{C}$, and the mixture was stirred at room temperature for 2.5 hours. The reaction was quenched with $\mathrm{H}_{2} \mathrm{O}$, and the resulting mixture was extracted with $\mathrm{CHCl}_{3}$. The organic extracts were washed with saturated aq $\mathrm{NH}_{4} \mathrm{Cl}$, saturated aq $\mathrm{NaHCO}_{3}$ and brine, dried over $\mathrm{Na}_{2} \mathrm{SO}_{4}$, filtered, and concentrated. The residue was purified by flash column chromatography on silica gel (hexane $/ \mathrm{EtOAc}=20 / 1$ to $1 / 1)$ to give $\mathbf{8}(56.5 \mathrm{mg}, 93 \%$ ) as a white solid. mp $142^{\circ} \mathrm{C} ;[\alpha]_{\mathrm{D}}^{22}+2.2\left(\mathrm{c} 1.0, \mathrm{CHCl}_{3}\right)$; IR $(\mathrm{KBr}) \mathrm{cm}^{-1}$; 3392, 3060, 2923, 1735, 1631, 1527, 1500, 1448, 1218, 761, 742, 700, 669, 617; ${ }^{1} \mathrm{H}-\mathrm{NMR}(270 \mathrm{MHz}$, $\left.\mathrm{CDCl}_{3}\right) \delta 7.76(2 \mathrm{H}, \mathrm{d}, J=7.3 \mathrm{~Hz}), 7.58(2 \mathrm{H}, \mathrm{d}, J=7.3 \mathrm{~Hz})$, $7.43 \sim 7.19(34 \mathrm{H}, \mathrm{m}), 6.96 \sim 6.90(2 \mathrm{H}, \mathrm{m}), 6.67(1 \mathrm{H}, \mathrm{d}$, $J=8.3 \mathrm{~Hz}), 5.82 \sim 5.66(2 \mathrm{H}, \mathrm{m}), 5.20 \sim 5.13(2 \mathrm{H}, \mathrm{m})$, $4.50 \sim 4.14(9 \mathrm{H}, \mathrm{m}), 3.91(1 \mathrm{H}, \mathrm{t}, J=7.1 \mathrm{~Hz}), 2.82(1 \mathrm{H}, \mathrm{dd}$, $J=8.1,12.8 \mathrm{~Hz}), 2.72(1 \mathrm{H}, \mathrm{dd}, J=7.9,12.8 \mathrm{~Hz}), 2.55(2 \mathrm{H}$, $\mathrm{dt}, J=4.6,12.0 \mathrm{~Hz}), 2.22 \sim 2.07(1 \mathrm{H}, \mathrm{m}), 1.65 \sim 1.42(6 \mathrm{H}$, m), $0.97 \sim 0.77(18 \mathrm{H}, \mathrm{m}) ;{ }^{13} \mathrm{C}-\mathrm{NMR}\left(67.2 \mathrm{MHz}, \mathrm{CDCl}_{3}\right) \delta$ $172.5,172.1,171.8,170.3,169.5,156.5,144.5,144.2$,
$144.1,143.7,143.6,141.1,131.1,129.5,129.4,129.3$, $128.4,127.9,127.8,127.5,127.0,126.7,125.0,119.8$, $118.3,67.0,66.9,66.8,65.9,52.6,52.1,51.7,51.2,46.9$, $39.5,39.3,38.6,33.5,33.3,30.3,24.6,24.5,22.8,22.7$, 22.0, 21.7, 19.2, 18.1; MS (FAB) $\mathrm{m} / z 1296.5887[\mathrm{M}+\mathrm{H}]^{+}$ (calcd for $\mathrm{C}_{79} \mathrm{H}_{86} \mathrm{O}_{8} \mathrm{~N}_{5} \mathrm{~S}_{2}: 1296.5918[\mathrm{M}+\mathrm{H}]$ ).

\section{Allyl L-Valinyl-D-leucinyl-L-leucinyl-D- $S$-tritylcysteinyl- D-S-tritylcysteinate}

A mixture of $8(52.4 \mathrm{mg}, 404 \mu \mathrm{mol})$ in piperidine $/ \mathrm{CH}_{2} \mathrm{Cl}_{2}$ $(1 / 4,0.80 \mathrm{ml})$ was stirred at $0^{\circ} \mathrm{C}$ for 1 hour. The reaction was quenched with $\mathrm{H}_{2} \mathrm{O}$, and the resulting mixture was extracted with $\mathrm{CHCl}_{3}$. The organic extracts were washed with saturated aq $\mathrm{NH}_{4} \mathrm{Cl}$ and brine, dried over $\mathrm{Na}_{2} \mathrm{SO}_{4}$, filtered, and concentrated. The residue was purified by flash column chromatography on silica gel $\left(\mathrm{CHCl}_{3}\right.$ to $\left.\mathrm{CHCl}_{3} / \mathrm{MeOH}=50 / 1\right)$ to give the amine $(38.7 \mathrm{mg}, 89 \%)$ as a white solid. mp $193 \sim 195^{\circ} \mathrm{C} ;[\alpha]_{\mathrm{D}}^{26}-7.6\left(\mathrm{c} 1.0, \mathrm{CHCl}_{3}\right)$; IR $(\mathrm{KBr}) \mathrm{cm}^{-1}$; 3262, 3054, 2956, 1747, 1633, 1538, 1442, $1170,742,692 ;{ }^{1} \mathrm{H}-\mathrm{NMR}\left(270 \mathrm{MHz}, \mathrm{CDCl}_{3}\right) \delta 7.62(1 \mathrm{H}, \mathrm{d}$, $J=4.2 \mathrm{~Hz}), 7.42 \sim 7.18(30 \mathrm{H}, \mathrm{m}), 6.99 \sim 6.90(3 \mathrm{H}, \mathrm{m})$, $5.85 \sim 5.71(1 \mathrm{H}, \mathrm{m}), 5.24 \sim 5.12(2 \mathrm{H}, \mathrm{m}), 4.51 \sim 4.23(6 \mathrm{H}$, $\mathrm{m}), 4.04(1 \mathrm{H}, \mathrm{d}, J=5.0 \mathrm{~Hz}), 3.05(1 \mathrm{H}, \mathrm{s}), 2.84(1 \mathrm{H}, \mathrm{dd}$, $J=8.7,12.5 \mathrm{~Hz}), 2.67(1 \mathrm{H}, \mathrm{dd}, J=6.9,12.5 \mathrm{~Hz}), 2.56(1 \mathrm{H}$, $\mathrm{dd}, J=4.6,13.4 \mathrm{~Hz}), 2.48(1 \mathrm{H}, \mathrm{dd}, J=4.6,12.9 \mathrm{~Hz})$, $1.75 \sim 1.44(7 \mathrm{H}, \mathrm{m}), \quad 0.92 \sim 0.81(18 \mathrm{H}, \mathrm{m}) ;{ }^{13} \mathrm{C}-\mathrm{NMR}$ $\left(67.2 \mathrm{MHz}, \mathrm{CDCl}_{3}\right) \delta 175.9,172.6,171.6,170.5,169.9$, $144.4,144.2,131.2,129.5,129.4,127.9,126.7,118.6$, 67.0, 66.7, 66.0, 60.0, 53.0, 51.8, 51.7, 51.2, 39.6, 39.4, 33.7, 33.1, 30.9, 24.7, 24.6, 23.0, 22.7, 22.0, 21.7, 19.6, 16.2; MS (FAB) $m / z \quad 1074.5270 \quad[\mathrm{M}+\mathrm{H}]^{+}$(calcd for $\left.\mathrm{C}_{64} \mathrm{H}_{76} \mathrm{O}_{6} \mathrm{~N}_{5} \mathrm{~S}_{2}: 1074.5237[\mathrm{M}+\mathrm{H}]\right)$.

\section{L-Valinyl-D-leucinyl-L-leucinyl-D-S-tritylcysteinyl-D-S- tritylcysteine (3)}

To a solution of the amine $(38.7 \mathrm{mg}, 360 \mu \mathrm{mol})$ in $\mathrm{THF} / \mathrm{H}_{2} \mathrm{O}(4 / 1,0.72 \mathrm{ml})$ was added $\mathrm{NaOH}(2.16 \mathrm{mg}$, $540 \mu \mathrm{mol})$ at $0^{\circ} \mathrm{C}$, and the mixture was stirred at $0^{\circ} \mathrm{C}$ for 1 hour. The reaction temperature was warmed to room temperature. After stirring at the same temperature for 5 hours, the reaction was quenched with $0.1 \mathrm{~N} \mathrm{HCl}(540 \mu 1)$, and the resulting mixture was extracted with $\mathrm{CHCl}_{3}$. The organic extracts were concentrated, and the residue was purified by flash column chromatography on silica gel $\left(\mathrm{CHCl}_{3}\right.$ to $\left.\mathrm{CHCl}_{3} / \mathrm{MeOH}=10 / 1\right)$ to give $3(34.0 \mathrm{mg}, 91 \%)$ as a white solid. $\mathrm{mp} 158 \sim 164^{\circ} \mathrm{C} ;[\alpha]_{\mathrm{D}}^{26}+3.7$ (c 1.0, $\left.\mathrm{CHCl}_{3}\right)$; IR (KBr) $\mathrm{cm}^{-1}$; 3297, 3058, 1958, 1731, 1660, 1531, 1492, 1444, 1241, 742, 700; ${ }^{1} \mathrm{H}-\mathrm{NMR}(270 \mathrm{MHz}$, Pyridine- $\left.d_{5}\right) \delta 10.5(1 \mathrm{H}, \mathrm{d}, J=8.3 \mathrm{~Hz}), 9.77(1 \mathrm{H}, \mathrm{d}$, $J=8.0 \mathrm{~Hz}), 9.17(1 \mathrm{H}, \mathrm{d}, J=7.6 \mathrm{~Hz}), 8.57(1 \mathrm{H}, \mathrm{d}, J=7.5 \mathrm{~Hz})$, $7.67 \sim 7.17(30 \mathrm{H}, \mathrm{m}), 5.27 \sim 4.91(3 \mathrm{H}, \mathrm{m}), 4.82(1 \mathrm{H}, \mathrm{dd}$, 
$J=6.9,13.5 \mathrm{~Hz}), 4.25(1 \mathrm{H}, \mathrm{dd}, J=6.3,11.6 \mathrm{~Hz}), 3.40(1 \mathrm{H}$, dd, $J=3.8,12.0 \mathrm{~Hz}), 3.27 \sim 2.94(3 \mathrm{H}, \mathrm{m}), 2.51 \sim 2.42(1 \mathrm{H}$, $\mathrm{m}), 2.14 \sim 2.01(2 \mathrm{H}, \mathrm{m}), 1.96 \sim 1.79(4 \mathrm{H}, \mathrm{m}), 1.21 \sim 0.86$ $(18 \mathrm{H}, \mathrm{m}) ;{ }^{13} \mathrm{C}-\mathrm{NMR}\left(67.2 \mathrm{MHz}\right.$, Pyridine- $\left.d_{5}\right) \delta 175.0$, $173.8,173.4,169.9,169.7,145.8,145.7,145.6,145.3$, $143.7,130.2,130.1,130.0,129.9,128.8,128.6,128.4$, $128.3,128.2,128.0,127.1,127.0,126.8,123.9,123.5$, 123.1, 66.9, 66.4, 64.2, 59.6, 54.4, 54.1, 52.1, 42.3, 40.7, 31.0, 25.8, 25.2, 23.1, 23.0, 22.9, 22.5, 19.0, 18.9; MS (FAB) $m / z 1034.4921[\mathrm{M}+\mathrm{H}]^{+}$(calcd for $\mathrm{C}_{61} \mathrm{H}_{72} \mathrm{O}_{6} \mathrm{~N}_{5} \mathrm{~S}_{2}$ : $1034.4924[\mathrm{M}+\mathrm{H}])$.

\section{Cyclo(-L-valinyl-D-leucinyl-L-leucinyl-D-S -tritylcysteinyl- D-S-tritylcysteinyl-)}

To a solution of $\mathbf{3}(100.0 \mathrm{mg}, 96.7 \mu \mathrm{mol})$ in $\mathrm{CH}_{2} \mathrm{Cl}_{2}(97 \mathrm{ml})$ were added NMM $(64.8 \mu 1,580 \mu \mathrm{mol})$, HOAt $(39.5 \mathrm{mg}$, $290 \mu \mathrm{mol})$ and HATU $(110.3 \mathrm{mg}, 290 \mu \mathrm{mol})$, and then the mixture was stirred at $0^{\circ} \mathrm{C}$ for 7 hours. The resulting mixture was concentrated, and the residue was purified by flash column chromatography on silica gel $\left(\mathrm{CHCl}_{3}\right.$ to $\left.\mathrm{CHCl}_{3} / \mathrm{MeOH}=200 / 1\right)$ to give the cyclicpentapeptide $(67.6 \mathrm{mg}, 69 \%)$ as a white solid. $\mathrm{mp}>300^{\circ} \mathrm{C} ;[\alpha]_{\mathrm{D}}^{21}-10.1$ (c 2.0, $\left.\mathrm{CHCl}_{3} / \mathrm{MeOH}=10 / 1\right)$; IR $(\mathrm{KBr}) \mathrm{cm}^{-1} ; 3268,3054$, 2956, 1639, 1538, 744, 698; ${ }^{1} \mathrm{H}-\mathrm{NMR}(270 \mathrm{MHz}$, Pyridine$\left.d_{5}\right) \delta 10.1(1 \mathrm{H}, \mathrm{d}, J=8.6 \mathrm{~Hz}), 10.0(1 \mathrm{H}, \mathrm{d}, J=6.3 \mathrm{~Hz}), 9.89$ $(1 \mathrm{H}, \mathrm{d}, J=8.6 \mathrm{~Hz}), 8.70(1 \mathrm{H}, \mathrm{d}, J=7.2 \mathrm{~Hz}), 8.05(1 \mathrm{H}, \mathrm{d}$, $J=9.6 \mathrm{~Hz}), 7.68 \sim 7.51(12 \mathrm{H}, \mathrm{m}), 7.29 \sim 7.16(18 \mathrm{H}, \mathrm{m}), 5.01$ $(1 \mathrm{H}, \mathrm{dd}, J=8.7,15.7 \mathrm{~Hz}), 4.89(1 \mathrm{H}, \mathrm{dt}, J=4.2,9.4 \mathrm{~Hz})$, $4.79 \sim 4.69(2 \mathrm{H}, \mathrm{m}), 4.63(1 \mathrm{H}, \mathrm{t}, J=9.6 \mathrm{~Hz}), 3.84(1 \mathrm{H}, \mathrm{dd}$, $J=9.1,11.8 \mathrm{~Hz}), 2.97(1 \mathrm{H}, \mathrm{dd}, J=4.6,12.2 \mathrm{~Hz}), 2.73 \sim 2.61$ $(2 \mathrm{H}, \mathrm{m}), 2.39(1 \mathrm{H}, \mathrm{dq}, J=6.9,12.7 \mathrm{~Hz}), 2.03 \sim 1.91(2 \mathrm{H}$, m), $1.83 \sim 1.66(4 \mathrm{H}, \mathrm{m}), 1.03(3 \mathrm{H}, \mathrm{d}, J=6.6 \mathrm{~Hz}), 1.00(3 \mathrm{H}$, d, $J=6.6 \mathrm{~Hz}), 0.93(3 \mathrm{H}, \mathrm{d}, J=6.3 \mathrm{~Hz}), 0.84(3 \mathrm{H}, \mathrm{d}$, $J=6.6 \mathrm{~Hz}), 0.80(3 \mathrm{H}, \mathrm{d}, J=6.3 \mathrm{~Hz}), 0.76(3 \mathrm{H}, \mathrm{d}, J=6.3 \mathrm{~Hz})$; ${ }^{13} \mathrm{C}-\mathrm{NMR}$ (67.2 MHz, Pyridine- $d_{5}$ ) $\delta$ 175.1, 173.3, 172.4, $170.7,169.9,145.5,145.1,130.0,129.7,129.0,128.3$, $128.2,127.0,126.8,67.0,66.6,59.7,54.0,53.8,53.3,51.7$, $41.1,39.7,34.1,33.2,27.6,25.0,24.9,22.8,22.7,22.2$, 21.6, 19.9, 18.9; MS (FAB) $m / z 1038.4683[\mathrm{M}+\mathrm{Na}]^{+}$ (calcd for $\mathrm{C}_{61} \mathrm{H}_{69} \mathrm{O}_{5} \mathrm{~N}_{5} \mathrm{~S}_{2} \mathrm{Na}$ : $1038.4638[\mathrm{M}+\mathrm{Na}]$ ).

\section{Malformin C}

A solution of $\mathrm{I}_{2}(45.5 \mathrm{mg}, 179 \mu \mathrm{mol})$ in DMF $(3.0 \mathrm{ml})$ was added to a solution of the cyclic pentapeptide $(60.8 \mathrm{mg}$, $59.8 \mu \mathrm{mol})$ in DMF $(3.0 \mathrm{ml})$ at room temperature. After stirring at room temperature for 30 minutes, $1.0 \%$ aq $\mathrm{Na}_{2} \mathrm{~S}_{2} \mathrm{O}_{3}$ was added to the reaction mixture which was then extracted with EtOAc. The organic extracts were washed with $\mathrm{H}_{2} \mathrm{O}$, dried over $\mathrm{Na}_{2} \mathrm{SO}_{4}$, filtered, and concentrated. The residue was treated with TFA $/ \mathrm{CH}_{2} \mathrm{Cl}_{2}(1 / 1,6.0 \mathrm{ml})$ and triisopropylsilane $(49.0 \mu \mathrm{l}, 239 \mu \mathrm{mol})$, the resulting mixture was stirred at room temperature for 1 hour. After concentration, the residue was purified by flash column chromatography on silica gel $\left(\mathrm{CHCl}_{3}\right.$ to $\left.\mathrm{CHCl}_{3} / \mathrm{MeOH}=30 / 1\right)$ to give malformin $\mathrm{C}(27.0 \mathrm{mg}, 85 \%)$ as a white solid. $\mathrm{mp}>300^{\circ} \mathrm{C} ;[\alpha]_{\mathrm{D}}^{22}-24.8$ (c 1.0, $\left.\mathrm{CHCl}_{3} / \mathrm{MeOH}=10 / 1\right)$; IR (KBr) cm ${ }^{-1} ; 3432,2962,1648$, 1536, 1201; ${ }^{1} \mathrm{H}-\mathrm{NMR}\left(270 \mathrm{MHz}, 1\right.$ drop $d$-TFA in $\left.\mathrm{CDCl}_{3}\right) \delta$ $5.10(1 \mathrm{H}, \mathrm{dd}, J=5.3,9.9 \mathrm{~Hz}), 4.49(1 \mathrm{H}, \mathrm{t}, J=3.0 \mathrm{~Hz}), 4.41$ $(1 \mathrm{H}, \mathrm{dt}, J=8.4,6.3 \mathrm{~Hz}), 4.11 \sim 4.05(2 \mathrm{H}, \mathrm{m}), 3.84(1 \mathrm{H}, \mathrm{dd}$, $J=3.0,15.5 \mathrm{~Hz}), 3.19 \sim 3.31(3 \mathrm{H}, \mathrm{m}), 2.03(1 \mathrm{H}, \mathrm{dt}, J=6.6$, $11.2 \mathrm{~Hz}), 1.74 \sim 1.49(6 \mathrm{H}, \mathrm{m}), 1.00 \sim 0.89(18 \mathrm{H}, \mathrm{m})$; ${ }^{13} \mathrm{C}$-NMR (67.2 MHz, 1 drop $d$-TFA in $\mathrm{CDCl}_{3}$ ) $\delta 177.1$, 176.5, 173.2, 171.3, 61.1, 54.6, 53.8, 53.4, 53.2, 46.8, 45.7, $38.7,38.6,27.5,25.0,24.7,22.1,22.0,21.5,21.2,18.8$, 18.1; MS (FAB) $\mathrm{m} / \mathrm{z} 530.2468 \quad[\mathrm{M}+\mathrm{H}]^{+}$(calcd for $\left.\mathrm{C}_{23} \mathrm{H}_{40} \mathrm{O}_{5} \mathrm{~N}_{5} \mathrm{~S}_{2}: 530.2471[\mathrm{M}+\mathrm{H}]\right)$.

Acknowledgement This work was supported by a grant from the 21st Century COE Program, Ministry of Education, Culture, Sports, Science and Technology. We also thank Ms. A. Nakagawa, Ms. C. Sakabe, and Ms. N. Sato (School of Pharmacy, Kitasato University) for the various instrumental analyses.

\section{References}

1. Hartwell LH, Kastan MB. Cell cycle control and cancer. Science 266: 1821-1828 (1994)

2. Hagimori K, Fukuda T, Hasegawa Y, Ōmura S, Tomoda H. Fungal malformins inhibit bleomycin-induced G2 checkpoint in Jurkat cells. Biol Pharm Bull 30: 1379-1383 (2007)

3. Curtis RW. Root curvatures induced by culture filtrate of Aspergillus niger. Science 128: 661-662 (1958)

4. Curtis RW. Curvatures and malformations in bean plants caused by culture filtrate of Aspergillus niger. Plant Physiol 33: 17-22 (1958)

5. Suda S, Curtis RW. Antibiotic properties of malformin. Appl Microbiol 14: 475-476 (1966)

6. Koizumi Y, Hasumi K. Enhancement of fibrinolytic activity of U937 cells by malformin A1. J Antibiot 55: 78-82 (2002)

7. Bodanszky M, Stahl GL. Structure and synthesis of malformin A. Proc Natl Acad Sci USA 71: 2791-2794 (1974) 